\title{
The association between type $D$ personality, and depression and anxiety ten years after PCI
}

\author{
M.N.A. AL-Qezweny ${ }^{1}$ E.M.W.J. Utens ${ }^{2} \cdot$ K. Dulfer ${ }^{2}$ B.A.F. Hazemeijer ${ }^{1}$ R-J. van Geuns ${ }^{1} \cdot$ J. Daemen $^{1}$ \\ R. van Domburg ${ }^{1}$
}

Published online: 13 June 2016

(c) The Author(s) 2016. This article is available at SpringerLink with Open Access

\begin{abstract}
Objective There are indications that type D personality and depression are associated in patients treated with percutaneous coronary intervention (PCI). However, at present it is unclear whether this relationship holds in the long term. This study's aim was to investigate the association between type $\mathrm{D}$ personality at 6 months post-PCI (baseline), and depression at 10-year follow-up. A secondary aim was to test the association between type D personality at baseline and anxiety at 10-year follow-up.

Methods A cohort of surviving consecutive patients $(N=$ 534) who underwent PCI between October 2001 and October 2002. Patients completed the type D personality scale (DS14) measuring type D personality at baseline, and the Hospital Anxiety and Depression Scale (HADS) measuring anxiety and depression at baseline and at 10 years post-PCI. Results At baseline, the prevalence of type D personality was $25 \%$ (135/534). Type D personality patients were more often depressed (42\%) than non-type D personality patients $(9 \%)$. Response rate of anxiety and depression questionnaires at 10 years was $75 \%$. At 10-year follow-up, $31 \%$ of type D personality patients were depressed versus $13 \%$ of non-type D personality patients. After adjustments, baseline type D personality remained independently associated with depression at 10 years $(\mathrm{OR}=3.69 ; 95 \% \mathrm{CI}$ [1.89-7.19]). Type D showed a similar association with
\end{abstract}

R. van Domburg

r.vandomburg@erasmusmc.nl

1 Department of Cardiology, Thoraxcenter, Erasmus Medical Center Rotterdam, Rotterdam, The Netherlands

2 Department of Child and Adolescent Psychiatry/Psychology, Erasmus MC - Sophia Children's Hospital, Rotterdam, The Netherlands anxiety at 10 years, albeit somewhat lower $(\mathrm{OR}=2.72$; $95 \%$ CI [1.31-5.63]).

Conclusions PCI patients with type D personality had a 3.69-fold increased risk for depression and a 2.72-fold increased risk for anxiety at 10 years of follow-up.

Keywords Depression - Anxiety · Coronary artery disease $\cdot$ PCI $\cdot$ Type D personality

\section{Introduction}

Since the introduction of drug-eluting stents in the early $2000 \mathrm{~s}$, prognosis has been improved significantly [1]. Therefore, secondary outcomes such as quality of life, safety, depression and anxiety have become more important.

Personality may play a role in the mental and physical health of coronary artery disease (CAD) patients. Patients with distressed personality (type D) showed negative affectivity and social inhibition [2] and this was associated with depression and anxiety [3, 4]. Moreover type D personality corresponded with adverse cardiac outcomes in patients with CAD. One study compared high negativity/high inhibition patients (type D) with high negativity/low inhibition patients. Type D personality showed an increased risk in major adverse cardiac events such as myocardial infarction (MI), percutaneous coronary intervention (PCI), and coronary artery bypass graft (CABG) at 15 months post-PCI while high negativity/low inhibition did not [5]. These are concerning findings since the prevalence of type $\mathrm{D}$ personality is around $20-50 \%$ and therefore type $\mathrm{D}$ is a factor that should not be ignored [6].

The prevalence of depression in patients with coronary artery disease (CAD) ranges from $25 \%$ to $50 \%$ [7-11]. 


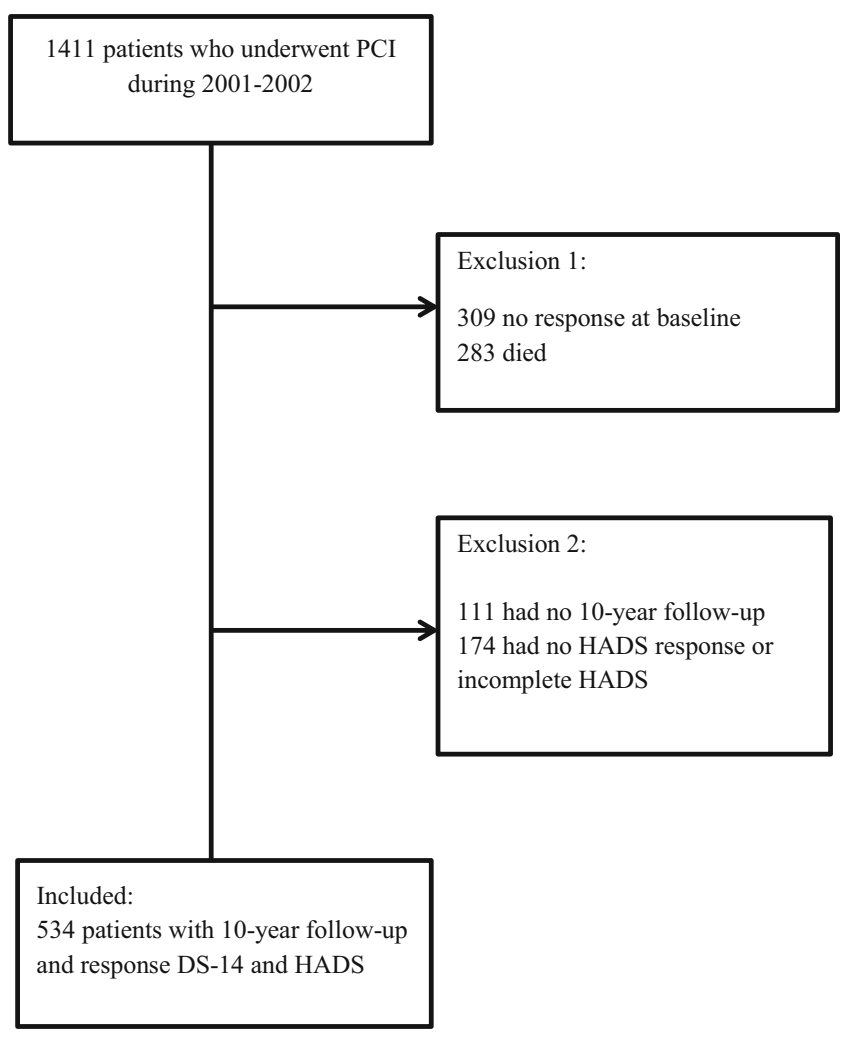

Fig. 1 Flowchart of patient inclusion

Studies have shown an increased mortality in patients with depression. The study populations consisted of patients with CAD, CABG, PCI and heart failure [12-16]. Depressed patients may have an increased risk of adverse events after PCI [16]. Depressed patients have a poorer prognosis with higher cardiac mortality compared with nondepressed patients [17].

Because recognition of depression might be too late, since the patient is then already depressed, early recognition of type D personality could improve treatment outcomes and lead to a better prognosis, since type $\mathrm{D}$ was associated with an increased risk for MI, PCI, CABG and adverse prognosis [5, 18]. With timely identification of type D personality, the risks of this personality style for (cardiac) health outcomes can be communicated with the patients and, if necessary, adequate referral to a mental health professional with expertise in the field can take place.

The aim of the present study was to investigate whether type D personality, assessed at baseline, is associated with depression, not only at one year post-PCI, but also at 10 years after PCI. Anxiety at 10-year follow-up was also investigated as a secondary objective.

\section{Methods}

\section{Participants and procedures}

This was a consecutive prospective cohort registry of patients who underwent PCI between October 2001 and October $2002(n=1411)$. At 6 months post-PCI (referred to as baseline in the remainder of this paper) patients were asked to fill in psychological questionnaires to assess anxiety, depression (using the Hospital Anxiety and Depression Scale; HADS), and type D personality (using the Type D Scale-14, DS14). Assessment of 6 months post-PCI was chosen to ascertain a stable medical and mental condition. At 10 years, survival status was assessed using the civil registry. All surviving patients were sent a letter including the HADS questionnaire.

Of the 1411 eligible patients who underwent PCI, 309 patients did not return the questionnaire at baseline. Of the remaining 1102 patients, 283 died. Of the 819 remaining patients, 534 responded to the HADS questionnaire (Fig. 1).

This study was not subject to the Dutch Medical Research Involving Human Subjects Act. Therefore, approval from the local research ethics committee to conduct this prospective follow-up study was not required at the time of enrolment. All patients consented to participation in this study by returning the questionnaire. However, in 2013 we received approval from our local medical ethics committee for this on-going PCI Registry (MEC-2013-262). The study was conducted according to the Helsinki Declaration [19].

\section{Sociodemographic and clinical characteristics}

Sociodemographic characteristics included gender and age. Clinical characteristics included multi-vessel disease (multi-vessel disease versus single-vessel disease/no vessel disease), cardiac history (i. e., previous MI, CABG surgery, or PCI), indication for PCI (angina pectoris, unstable angina pectoris, or MI), cardiac risk factors (i. e., hypertension, hypercholesterolaemia, diabetes, family history of CAD and self-reported smoking). Information on clinical variables was prospectively collected at the time of the index$\mathrm{PCI} /$ baseline and recorded in the institutional database.

\section{Type D personality}

The DS14 was used to assess type D personality. The DS14 uses two subscales with seven items each. One scale is used to assess negative affectivity and the other one is used to assess social inhibition. Negative affectivity is a term that covers certain feelings such as 'I often feel unhappy' and 'I often find myself worrying about something'. While social inhibition covers social discomfort and problems with social interactions [20]. Items can be scored from 0 (false) to 
Tab. 1 Baseline characteristics according to type D and non-type D

\begin{tabular}{|c|c|c|c|}
\hline & Type D & Non-Type D & $P$ \\
\hline No. of patients & $135(25 \%)$ & $399(75 \%)$ & \\
\hline Male & $96(71 \%)$ & $305(77 \%)$ & 0.24 \\
\hline Age $\pm S D$ & $59 \pm 9.7$ & $60 \pm 9.6$ & 0.19 \\
\hline \multicolumn{4}{|l|}{ Clinical characteristics } \\
\hline Multivessel disease & $64(47 \%)$ & $185(46 \%)$ & 0.83 \\
\hline Old MI & $50(37 \%)$ & $145(37 \%)$ & 0.98 \\
\hline Old PCI & $17(13 \%)$ & $75(19 \%)$ & 0.10 \\
\hline Old CABG & $12(9 \%)$ & $32(8 \%)$ & 0.75 \\
\hline \multicolumn{4}{|l|}{ Risk factors } \\
\hline Hypertension & $53(39 \%)$ & $138(35 \%)$ & 0.33 \\
\hline Family history & $50(37 \%)$ & $121(30 \%)$ & 0.15 \\
\hline Current smoker & $57(42 \%)$ & $123(31 \%)$ & 0.02 \\
\hline Diabetes mellitus & $18(13 \%)$ & $38(10 \%)$ & 0.21 \\
\hline Cholesterol & $111(82 \%)$ & $324(81 \%)$ & 0.79 \\
\hline Indication for PCI & & & 0.29 \\
\hline Stable angina & $59(44 \%)$ & $199(50 \%)$ & \\
\hline Unstable angina & $54(40 \%)$ & $130(33 \%)$ & \\
\hline Acute myocardial infarction & $22(16 \%)$ & $70(18 \%)$ & \\
\hline \multicolumn{4}{|l|}{ Medication } \\
\hline Aspirin & $130(96 \%)$ & $389(98 \%)$ & 0.36 \\
\hline Calcium antagonist & $72(53 \%)$ & $189(48 \%)$ & 0.24 \\
\hline Beta blocker & $90(67 \%)$ & $273(69 \%)$ & 0.68 \\
\hline Oral nitrates & $17(13 \%)$ & $54(14 \%)$ & 0.77 \\
\hline Diuretic & $21(16 \%)$ & $51(13 \%)$ & 0.42 \\
\hline ACE inhibitor & $33(24 \%)$ & $128(32 \%)$ & 0.09 \\
\hline Statin & $108(80 \%)$ & $312(78 \%)$ & 0.69 \\
\hline \multicolumn{4}{|l|}{ Psychological characteristics } \\
\hline Anxiety & $73(54 \%)$ & $61(15 \%)$ & $<0.001$ \\
\hline Depression & $57(42 \%)$ & $35(9 \%)$ & $<0.001$ \\
\hline
\end{tabular}

4 (true). Type D personality was identified if both subscales scored $\geq 10$ points [21].

\section{Anxiety and depression}

Anxiety and depression was assessed by completing the Dutch version of the HADS. The HADS consists of two subscales. One subscale is used to measure anxiety and the other is used to measure depression. Each subscale has seven items which can be scored from 0 to 3 . A cut-off score of $\geq 8$ was used to determine depression and anxiety [22].

\section{Statistical analysis}

Patients were grouped according to type D and non-type D personality. Differences between groups were analysed using the Chi-square test for nominal variables and the Student's t-test for continuous variables. A multivariable logistic regression model was used to investigate the association between type D personality and depression and anxiety, respectively. Also adjustments were made for all baseline characteristics. Additionally, adjustments were made for baseline anxiety and depression. Interactions between type D personality, and anxiety and depression were investigated.

The outcomes were reported with odds ratio (OR) and their corresponding $95 \%$ confidence interval (CI). All results were based on two-tailed tests and a $p<0.05$ was used to indicate statistical significance. Data analysis has been done with SPSS version 21.

\section{Results}

\section{Baseline characteristics}

The prevalence of baseline type D personality was $25 \%$ $(135 / 534)$. The prevalence of depression at baseline was $42 \%(57 / 135)$ in type D personality patients versus $9 \%$ 
Tab. 2 Univariable and multivariable analysis: association between baseline type D and depression and anxiety at 10 years

\begin{tabular}{|c|c|}
\hline & OR $[95 \% \mathrm{CI}]$ \\
\hline \multicolumn{2}{|l|}{ Depression } \\
\hline Univariable type D personality & $3.08[1.93-4.92]$ \\
\hline Adjusted for baseline characteristics type D personality ${ }^{a}$ & $3.70[2.23-6.13]$ \\
\hline Adjusted for baseline characteristics + baseline depression type D personality & $3.69[1.89-7.19]$ \\
\hline \multicolumn{2}{|l|}{ Anxiety } \\
\hline Univariable type D personality & $3.43[2.22-5.30]$ \\
\hline Adjusted for baseline characteristics type D personality ${ }^{a}$ & $3.52[2.24-5.53]$ \\
\hline Adjusted for baseline characteristics + baseline anxiety type D personality & $2.72[1.31-5.63]$ \\
\hline
\end{tabular}

${ }^{a}$ Excluding baseline depression/baseline anxiety

(35/399) in non-type D personality patients. Anxiety was also more prevalent in the type D personality patients. Furthermore, most baseline characteristics did not differ between the two groups, except for the finding that type D personality patients were more often smokers (Tab. 1).

\section{Association between baseline type D personality and depression at 10 years}

The prevalence of depression at 10 years of follow-up was $31 \%(42 / 135)$ in type D personality patients versus $13 \%$ (51/399) in non-type D personality patients. Univariable analysis showed a strong association between type D personality and depression at 10 years $(\mathrm{OR}=3.08 ; 95 \% \mathrm{CI}$ [1.93-4.92]) (Tab. 2). After adjusting for baseline characteristics, type D personality showed an even stronger association with depression $(\mathrm{OR}=3.70 ; 95 \% \mathrm{CI}$ [2.23-6.13]). In the third model, type D was adjusted for baseline characteristics and baseline depression. The OR remained the same but the CI widened (OR $=3.69 ; 95 \%$ CI [1.89-7.19]). Depression at baseline was found to be a strong cofactor and therefore an interaction variable was made. The interaction between type $\mathrm{D}$ personality and depression at baseline proved to be significant and, therefore, the interaction was added in the multivariable model. The analysis showed that the association between type D personality and depression was stable in all models (Tab. 2).

\section{Association between baseline type $D$ personality and anxiety at 10 years}

The prevalence of anxiety at 10-year follow-up was $40 \%(54 / 135)$ in type D personality patients versus $16 \%$ $(65 / 399)$ in non-type D personality patients. In univariable analysis type D personality was associated with anxiety at 10 -year follow-up $(\mathrm{OR}=3.43 ; 95 \% \mathrm{CI}[2.22-5.30])$ (Tab. 2). After adjusting for baseline characteristics, type D personality remained associated with anxiety $(\mathrm{OR}=3.52$; $95 \%$ [2.24-5.53]). In the third model, type D was adjusted for baseline characteristics and baseline anxiety. The OR between type D and anxiety at 10 years was still significant $(\mathrm{OR}=2.72 ; 95 \% \mathrm{CI}$ [1.31-5.63]). Anxiety at baseline was found to be a strong cofactor and therefore an interaction variable was made. The interaction between type D personality and anxiety proved to be significant and, therefore, the interaction was added in the multivariable model.

\section{Discussion}

Type D personality early after PCI was associated with a 3.69-fold increased risk for depression and 2.72-fold increased risk for anxiety 10 years after PCI. Earlier, our research group reported an association between type D personality and depressive symptoms at one year [3, 23]. This present study showed that this association sustained at least up to 10 years after PCI. Two other studies outside of our research group also showed an association between type D personality and depression with a follow-up period of 1 year [24, 25].

One study, however, found no relation between type D personality and the development of major depression or minor depression in 250 patients who had an acute coronary syndrome [26]. Major or minor depression in this study was measured by the DSM-IV. This raises questions whether type D personality is a personality trait or not. The limitation of that study was its small sample size. Furthermore, the HADS used in our study has been proven to be a valid questionnaire for symptom severity and case recognition of anxiety and depression disorder [22].

The patients selected for this study were a good representation of PCI patients. It should be noted that our patients were Dutch-speaking. The question can be raised whether type D personality generalises across regions and cultures. However, type D personality was shown to be generalised across cultures in a recent study [6]. Together with the findings of this study and the previous ones, it shows that type D personality is universal. Though the cross-cultural analysis of the study only extends to European and English- 
speaking countries, it may also extend to Asian countries $[27,28]$.

Despite all the evident results of the effect of type D personality, there still is a discussion concerning the validity of type D personality. Bacon et al. suggested in their commentary that while studies show that type D personality is independently associated with depression in different populations, the cut-off point of 10 was criticised [29].

The same statistical models were used to test associations between type D personality at baseline and depression and anxiety, respectively, at 10-year follow-up. Type D personality was proved to be not only associated with an increased risk for depression at 10 years of follow-up, but also with an increased risk for anxiety. Previously we reported the association between type $\mathrm{D}$ personality at baseline and anxiety at one year in PCI patients $[4,30]$. Now we have extended our findings regarding this association to up to 10 years of follow-up.

Unfortunately, systematic data regarding the use of psychotherapy or antidepressant or antianxiety medication in our patient sample were not available. Therefore, it is unknown to what extent these factors may have influenced the present findings. Future research should also take these treatments into account.

At baseline we achieved a response rate of $79 \%$, which is quite reasonable. During 10 years a number of patients died or were lost to follow-up and for the remaining patients we achieved a very similar result with a response rate of $75 \%$. While we are of the opinion that the numbers are reasonable, we should acknowledge that this could introduce selection bias.

In summary, type D personality is a predictive tool to identify patients who have an elevated risk for depression and anxiety, 10 years after PCI. Type D personality has been shown to be a stable personality taxonomy [4]. So, presumably, a patient with type $\mathrm{D}$ personality will have this personality for many years or for the rest of his or her life, making it more reliable than the HADS, which is a state measure fluctuating across time and situation. The importance of these results lies in the clinical use of type D personality in identifying PCI patients with a high risk for depression and anxiety.

\section{Funding sources None}

Conflict of interests We wish to confirm that there are no known conflicts of interest associated with this publication

Open Access This article is distributed under the terms of the Creative Commons Attribution 4.0 International License (http:// creativecommons.org/licenses/by/4.0/), which permits unrestricted use, distribution, and reproduction in any medium, provided you give appropriate credit to the original author(s) and the source, provide a link to the Creative Commons license, and indicate if changes were made.

\section{References}

1. Moses JW, Leon MB, Popma JJ, et al. Sirolimus-eluting stents versus standard stents in patients with stenosis in a native coronary artery. N Engl J Med. 2003;349:1315-23.

2. Denollet J. Type d personality. A potential risk factor refined. J Psychosom Res. 2000;49:255-66.

3. Pedersen SS, Denollet J, Gestel YR van, Serruys PW, Domburg RT van. Clustering of psychosocial risk factors enhances the risk of depressive symptoms 12-months post percutaneous coronary intervention. Eur J Cardiovasc Prev Rehabil. 2008;15:203-9.

4. Gestel YR van, Pedersen SS, Sande M van de, et al. Type-D personality and depressive symptoms predict anxiety 12 months postpercutaneous coronary intervention. J Affect Disord. 2007;103: 197-203.

5. Denollet J, Pedersen SS, Ong AT, Erdman RA, Serruys PW, Domburg RT van. Social inhibition modulates the effect of negative emotions on cardiac prognosis following percutaneous coronary intervention in the drug-eluting stent era. Eur Heart J. 2006;27:171-7.

6. Kupper N, Pedersen SS, Hofer S, Saner H, Oldridge N, Denollet J. Cross-cultural analysis of type D (distressed) personality in 6222 patients with ischemic heart disease: a study from the international heart QoL project. Int J Cardiol. 2013;166:327-33.

7. Lesperance F, Frasure-Smith N, Talajic M, Bourassa MG. Five-year risk of cardiac mortality in relation to initial severity and one-year changes in depression symptoms after myocardial infarction. Circulation. 2002;105:1049-53.

8. Burg MM, Benedetto MC, Soufer R. Depressive symptoms and mortality two years after coronary artery bypass graft surgery (CABG) in men. Psychosom Med. 2003;65:508-10.

9. Jiang W, Kuchibhatla M, Cuffe MS, et al. Prognostic value of anxiety and depression in patients with chronic heart failure. Circulation. 2004;110:3452-6.

10. Parakh K, Thombs BD, Fauerbach JA, Bush DE, Ziegelstein RC. Effect of depression on late (8 years) mortality after myocardial infarction. Am J Cardiol. 2008;101:602-6.

11. Connerney I, Sloan RP, Shapiro PA, Bagiella E, Seckman C. Depression is associated with increased mortality 10 years after coronary artery bypass surgery. Psychosom Med. 2010;72:874-81.

12. Damen NLMN, Versteeg H, Boersma E, Van Geuns RM, Van Domburg RT, Pedersen SS. Depression is independently associated with 7-year mortality in patients treated with percutaneous coronary intervention: results from the reseach registry. Eur J Cardiovasc Nurs. 2012;11:S5-S6.

13. Frasure-Smith N, Lesperance F, Gravel G, et al. Social support, depression, and mortality during the first year after myocardial infarction. Circulation. 2000;101:1919-24.

14. Jiang W, Kuchibhatla M, Clary GL, et al. Congestive heart failure relationship between depressive symptoms and long-term mortality in patients with heart failure. Am Heart J. 2007;154:102-8.

15. Denollet J, Martens EJ, Smith ORF, Burg MM. Efficient assessment of depressive symptoms and their prognostic value in myocardial infarction patients. J Affect Disord. 2010;120:105-11.

16. Pedersen SS, Denollet J, Daemen J, et al. Fatigue, depressive symptoms, and hopelessness as predictors of adverse clinical events following percutaneous coronary intervention with paclitaxel-eluting stents. J Psychosom Res. 2007;62:455-61.

17. Frasure-Smith N, Lesperance F, Talajic M. Depression and 18month prognosis after myocardial infarction. Circulation. 1995;91: 999-1005.

18. Pedersen SS, Denollet J. Is type D personality here to stay? Psychol Health. 2006;21:117.

19. Goodyear MD, Krleza-Jeric K, Lemmens T. The declaration of Helsinki. BMJ. 2007;335:624-5. 
20. Denollet J. DS14: standard assessment of negative affectivity, social inhibition, and type D personality. Psychosom Med. 2005;67:89-97.

21. Emons WHM, Meijer RR, Denollet J. Negative affectivity and social inhibition in cardiovascular disease: Evaluating type-D personality and its assessment using item response theory. J Psychosom Res. 2007;63:27-39.

22. Bjelland I, Dahl AA, Haug TT, Neckelmann D. The validity of the Hospital Anxiety and Depression Scale. An updated literature review. J Psychosom Res. 2002;52:69-77.

23. Pedersen SS, Ong AT, Sonnenschein K, Serruys PW, Erdman RA, Domburg RT van. Type D personality and diabetes predict the onset of depressive symptoms in patients after percutaneous coronary intervention. Am Heart J. 2006;151(367):e1-e6.

24. Christodoulou C, Douzenis A, Mommersteeg PM, et al. A casecontrol validation of Type D personality in Greek patients with stable coronary heart disease. Ann Gen Psychiatry. 2013;12:38.

25. Doyle F, McGee H, Delaney M, Motterlini N, Conroy R. Depressive vulnerabilities predict depression status and trajectories of de- pression over 1 year in persons with acute coronary syndrome. Gen Hosp Psychiatry. 2011;33:224-31.

26. Marchesi C, Ossola P, Scagnelli F, et al. Type D personality in never-depressed patients and the development of major and minor depression after acute coronary syndrome. J Affect Disord. 2014;155:194-9.

27. Yu DS, Thompson DR, Yu CM, Pedersen SS, Denollet J. Validating the type D personality construct in Chinese patients with coronary heart disease. J Psychosom Res. 2010;69:111-8.

28. Lim HE, Lee MS, Ko YH, et al. Assessment of the type D personality construct in the Korean population: a validation study of the Korean DS14. J Korean Med Sci. 2011;26:116-23.

29. Bacon SL, Moullec G. Type-D personality and heart disease: It might be 'one small step', but it is still moving forward: a comment on grande et al. Ann Behav Med. 2012;43:280-1.

30. Spindler H, Pedersen SS, Serruys PW, Erdman RA, Domburg RT van. Type-D personality predicts chronic anxiety following percutaneous coronary intervention in the drug-eluting stent era. J Affect Disord. 2007;99:173-9. 